\title{
Overexpression of Aquaporin-1 and Caveolin-1 in the Rat Urinary Bladder Urothelium Following Bladder Outlet Obstruction
}

\author{
Sun-Ouck Kim, Seung Hee Song, Kwangsung Park, Dongdeuk Kwon \\ Department of Urology, Chonnam National University Hospital, Chonnam National University Medical School, Gwangju, Korea
}

\begin{abstract}
Purpose: This study was designed to investigate the effect of detrusor overactivity induced by partial bladder outlet obstruction (BOO) on the expression of aquaporin 1 (AQP1) and caveolin 1 (CAV1) in the rat urinary bladder, and to determine the role of these molecules in detrusor overactivity.

Methods: Female Sprague-Dawley rats were divided into control $(n=30)$ and experimental $(n=30)$ groups. The BOO group underwent partial BOO, and the control group underwent a sham operation. After 4 weeks, an urodynamic study was performed to measure the contraction interval and contraction pressure. The expression and cellular localization of AQP1 and CAV1 were determined by western blot and immunofluorescence experiments in the rat urinary bladder.

Results: In cystometrograms, the contraction interval was significantly lower in the $\mathrm{BOO}$ group ( $2.9 \pm 1.5$ minutes) than in the control group $(6.7 \pm 1.0$ minutes $)(\mathrm{P}<0.05)$. Conversely, the average contraction pressure was significantly higher in the $\mathrm{BOO}$ group $(21.2 \pm 3.3 \mathrm{mmHg})$ than in the control group $(13.0 \pm 2.5 \mathrm{mmHg})(\mathrm{P}<0.05)$. AQP1 and CAV1 were coexpressed in the capillaries, arterioles, and venules of the suburothelial layer. AQP1 and CAV1 protein expression was significantly increased in the $\mathrm{BOO}$ rats compared to the control rats $(\mathrm{P}<0.05)$.

Conclusions: Detrusor overactivity induced by BOO causes a significant increase in the expression of AQP1 and CAV1, which were coexpressed in the suburothelial microvasculature. This finding suggests that AQP1 and CAV1 might be closely related to bladder signal activity and may have a functional role in BOO-associated detrusor overactivity.
\end{abstract}

Keywords: Aquaporin 1; Caveolin 1; Bladder outlet obstruction; Rats

\section{INTRODUCTION}

The urothelium has been considered a simple passive barrier between the urinary tract and urine. However, the urothelium has recently been found to be a responsive organ capable of sensing a variety of signals from the urinary bladder [1]. Studies have revealed that the urothelium can release chemical mediators, express a number of sensor molecules, and respond to external stimuli [2].

Bladder outlet obstruction (BOO) is associated with physiologic and morphologic changes, including impaired contractility and bladder smooth muscle hypertrophy [3]. Functional al- terations of the urinary bladder induced by $\mathrm{BOO}$ are associated with ultrastructural changes involving mitochondria and the plasma membrane [4].

The potential role of aquaporins (AQPs) in the functional alteration of the urinary bladder has been investigated [5-7]. AQPs are a family of transmembrane proteins that mediate water transport across cell membranes [8]. Aquaporin 1 (AQP1) is constitutively expressed in cells in which net water transport does not appear to be important, such as endothelial cells and vascular smooth muscle cells $[9,10]$.

Caveolae are small flask-shaped invaginations of the cell membrane that are thought to play an important role in cell surface
Corresponding author: Sun-Ouck Kim

Department of Urology, Chonnam National University Hospital, Chonnam National University Medical School, 42 Jebong-ro, Dong-gu, Gwangju 501-757, Korea

TEL: +82-62-220-6705 / FAX: +82-62-227-1643 / E-mail: seinsena@hanmail.net Submitted: November 2, 2013 / Accepted after revision: November 29, 2013
This is an Open Access article distributed under the terms of the Creative Commons Attribution Non-Commercial License (http://creativecommons.org/licenses/by-nc/3.0/) which permits unrestricted non-commercial use, distribution, and reproduction in any medium, provided the original work is properly cited. 
signaling [11]. Caveolins (CAVs), the integral membrane proteins of the caveolae, are essential for their formation. Among the three CAV isoforms, caveolin 1 (CAV1) has been shown to play a role in the regulation of signal transduction pathways in various endothelial and epithelial cells [12]. Functional roles of CAV1 are beginning to emerge from studies of CAV1 knockout mice. Mice lacking CAV1 also lack caveolae in the bladder and exhibit several urological disorders, including decreased excretion of acetylcholine and disruption of the M3 muscarinic cholinergic activity of the bladder [13].

In our previous report, altered expression of $\mathrm{AQPs}$ was observed in $\mathrm{BOO}$ urinary bladders showing detrusor overactivity $[7,14]$. Hormonal depletion induced by ovariectomy caused a significant change in CAV1 expression and detrusor activity, which was restored to the normal control value after hormonal replacement [15]. In the cyclophosphamide-induced cystitis rat model, CAV1 expression changed along with the inflammatory reaction of the urinary bladder [16]. However, to date, no assessment of the interrelationship between AQPs and CAVs in the urinary bladder has been reported. The purpose of this study was to investigate the effect of detrusor overactivity induced by $\mathrm{BOO}$ on the expression of $\mathrm{AQP} 1$ and CAV1 and to determine the role of these molecules in the detrusor overactivity that occurs in association with $\mathrm{BOO}$ in a rat model. We specifically focused on the suburothelium, an emerging research target area in bladder dysfunction.

\section{MATERIALS AND METHODS}

\section{Experimental Model}

Female Sprague-Dawley rats were divided into control $(n=30)$ and experimental $(n=30)$ groups. The experimental group underwent partial BOO. Animals were premedicated with xylazine $(2.2 \mathrm{mg} / \mathrm{kg}$, intramuscular) and were anesthetized with a zolazepam/tiletamine cocktail ( $4.4 \mathrm{mg} / \mathrm{kg}$, intramuscular). A midline abdominal incision was performed and the bladder and proximal urethra were dissected free of the surrounding tissue. To create an intravesical obstruction, a polyethylene catheter-90 was placed beside the proximal urethra and a 3-zero silk ligature was tied around the urethra and catheter. The catheter was subsequently removed and the abdominal incision was closed. The control group underwent a sham operation. After 4 weeks, urodynamic and histologic studies were performed. All rats were fed a standard diet until the day prior to the experiments. The rats were deprived of food overnight before the ex- periments were performed. The expression and cellular localization of AQP1 and CAV1 were determined by western blot and immunofluorescence experiments. The study was approved by the Ethics Committee of the Chonnam National University Medical School.

\section{Cystometrogram}

Four weeks after the operation, rats ( $\mathrm{n}=10$ in each group) were anesthetized with a subcutaneous injection of $1.2 \mathrm{~g} / \mathrm{kg}$ urethane. A suprapubic midline incision was performed to expose the bladder, and a transvesical catheter with a fire-flared tip (polyethylene catheter-50) was inserted into the dome of the bladder and secured with a ligature. The abdomen was then closed. The catheter was connected to a pressure transducer and syringe pump via a 3-way stopcock to record the intravesical pressure and to infuse saline into the bladder. After the bladder was emptied, cystometry was performed with saline infusion at a rate of $0.04 \mathrm{~mL} / \mathrm{min}$. The contraction pressure and contraction interval were recorded.

\section{Western Blot}

All minced bladder tissues were homogenized in ice-cold isolation solution with a Tissumizer homogenizer (Tekmar, Cincinnati, OH, USA). Tissues were homogenized using a microsawtooth generator, applying five bursts of five strokes. Tissue homogenates ( $\mathrm{n}=10$ in each group, $50 \mu \mathrm{g}$ of protein) were separated by $12 \%$ sodium dodecyl sulfate-polyacrylamide gel electrophoresis and transferred to polyvinylidene difluoride membranes (Amersham Pharmacia Biotech, Buckinghamshire, UK). The blots were then washed with Tris-buffered saline containing Tween-20 (TBS-T; 10 mM Tris-HCl, pH 7.6, 150 mM NaCl, $0.05 \%$ Tween-20). The membranes were blocked with $5 \%$ skimmed milk for 1 hour and incubated with the appropriate primary antibody. Monoclonal mouse antibodies against AQP1 (1:2,000; Chemicon, Temecula, CA, USA) and CAV1 (1:2,000; Chemicon), and a polyclonal rabbit antibody against glyceraldehyde 3-phosphate dehydrogenase (GAPDH) (1:4,000; Cell Signaling Technology, Danvers, MA, USA) were used. The membranes were then washed, and the proteins were detected using a goat anti-mouse-IgG (for CAV1) or a goat anti-rabbitIgG (for AQP1 and GAPDH) conjugated to horseradish peroxidase. Antibody incubations were performed in a $4^{\circ} \mathrm{C}$ incubator. The bands were visualized by enhanced chemiluminescence (Amersham Pharmacia Biotech). GAPDH was used as an internal control. Densitometry was performed using the Multi 
gauge V3.0 (Life-science, Fujifilm Global, Tokyo, Japan) chemiluminescence system and analysis software.

\section{Immunofluorescence Staining}

The bladder tissue sections ( $\mathrm{n}=10$ in each group, 10 sections for each animal) were rinsed in phosphate buffered saline (PBS) and then treated with normal chicken serum for 30 minutes to block nonspecific binding. After washing in PBS, the sections were incubated with antibodies for AQP1 (1:100; Chemicon) and CAV1 (1:100; Chemicon) in PBS for $12-14$ hours at $4^{\circ} \mathrm{C}$. Immunoreactivity for $\mathrm{CAV} 1$ and AQP1 was detected using Alexa Fluor 594-conjugated chicken anti-mouse-IgG $(\mathrm{H}+\mathrm{L})$ and Alexa Fluor 488-conjugated chicken anti-rabbit-IgG $(\mathrm{H}+\mathrm{L})$ (Molecular Probes Inc., Eugene, OR, USA), respectively. Tissue sections were mounted using a mounting solution containing 4'-6-diamidino-2-phenylindole. The negative control tissues were prepared in a similar manner, but the AQP1 and CAV1 antibodies were omitted from the incubation solution. The tissue sections were examined with an LSM 510 (Carl Zeiss, Seoul, Korea) confocal microscope with an excitation wavelength appropriate for the Alexa Fluor dyes (405, 488, and $594 \mathrm{~nm}$ ). Final images were constructed using the LSM Image Examiner software.

\section{Statistical Analysis}

The results are expressed as the mean \pm standard deviation, except the data for cystometric parameters, which are expressed as the mean \pm standard error of the mean. The Mann-Whitney test was used for statistical analysis, and tested the null hypothesis that no differences existed between the two groups in the mean expression levels of AQP1 and CAV1. Differences were considered significant at $\mathrm{P}<0.05$.

\section{RESULTS}

All of the animals survived for 3 weeks after partial BOO. There was no difference in body weight between the two groups. The weight of the urinary bladders was significantly higher in the BOO group $(610 \pm 18.1 \mathrm{mg})$ than in the control group (132.4 \pm $19.7 \mathrm{mg})(\mathrm{P}<0.05)$. This finding indicated that partial $\mathrm{BOO}$ was properly introduced.

\section{Effect of BOO on Cystometric Parameters}

In cystometrograms, the contraction interval was significantly lower in the BOO group ( $2.9 \pm 1.5$ minutes) than in the control
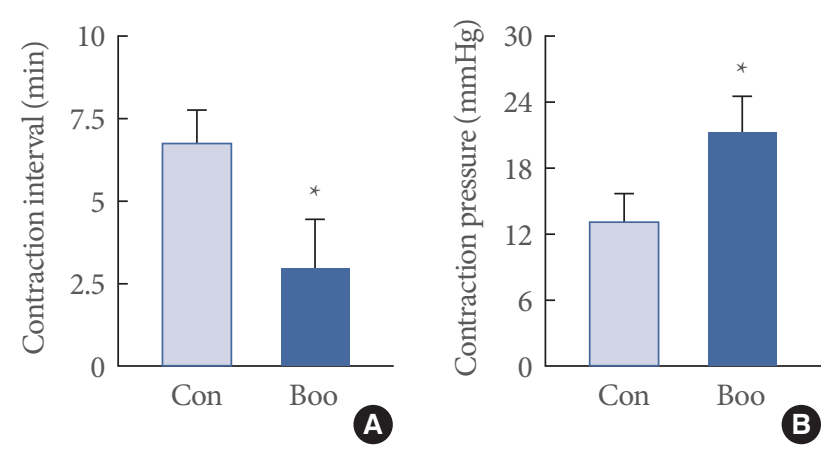

Fig. 1. Representative urodynamic profiles of control and bladder outlet obstruction (BOO) bladders. (A) The contraction interval was decreased significantly in the $\mathrm{BOO}$ group compared with the control $\left({ }^{\star} \mathrm{P}<0.05\right)$. (B) The average contraction pressure was also significantly increased in the BOO group compared with the control. The graph denotes the means \pm standard error of the mean of 10 experiments for each condition. ${ }^{\star} \mathrm{P}<0.05$ versus control. Con, control.

group $(6.7 \pm 1$ minutes $)(\mathrm{P}<0.05)$. Conversely, the average contraction pressure was significantly higher in the $\mathrm{BOO}$ group $(21.2 \pm 3.3 \mathrm{mmHg})$ than in the control group $(13.0 \pm 2.5 \mathrm{mmHg})$ $(\mathrm{P}<0.05)$ (Fig. 1).

\section{Effect of BOO on the Expression of AQP1 and CAV1}

AQP1 and CAV1 were coexpressed in the capillaries, venules, and arterioles of the suburothelial layer (Fig. 2). Immunofluorescence studies revealed that, in terms of the cellular patterns of labeling, the expression of AQP1 and CAV1 in the control group was similar to that in the BOO group (Fig. 2). For both groups, western blot analysis revealed bands at $28 \mathrm{kDa}$ and 22 $\mathrm{kDa}$ corresponding to the AQP1 and CAV1 proteins (Fig. 3). $\mathrm{AQP1}$ and CAV1 protein expression was significantly increased in the $\mathrm{BOO}$ rats compared to the controls $(\mathrm{P}<0.05)$ (Fig. 3).

\section{DISCUSSION}

This study demonstrated the coexpression of AQP1 and CAV1 in the suburothelial microvasculature and significantly increased expression of these proteins following $\mathrm{BOO}$ in a rat model. Cystometric results showed that the BOO bladder exhibited a decreased voiding interval and increased voiding pressure compared to the control bladder, indicating detrusor overactivity. Immunohistochemical experiments showed that AQP1 and CAV1 were colocalized in the urinary bladder, specifically in the capillaries, venules, and arterioles of the suburothelial layer. These results provide evidence that $\mathrm{BOO}$ induces bladder insta- 

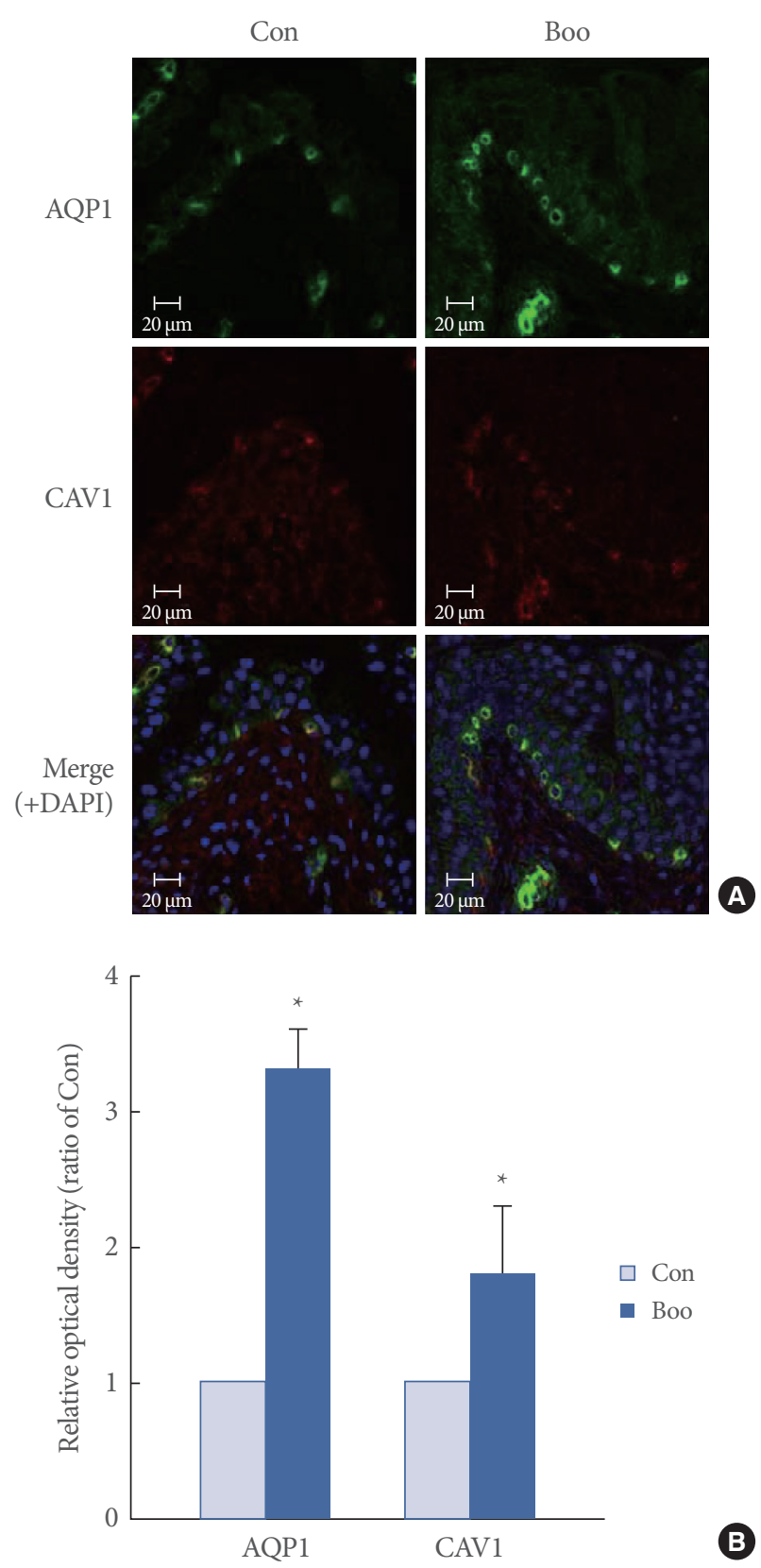

Fig. 2. (A) Immunofluorescence labeling of aquaporin 1 (AQP1) and caveolin 1 (CAV1) in the rat urinary bladder. AQP1 (green) and CAV1 (red) coexpression (merge) was noted throughout the capillaries, venules, and vascular smooth muscle. The expression of AQP1 and CAV1 was increased in the bladder outlet obstruction (BOO) group. The horizontal scale bar at the bottom left of each figure indicates the magnification power. (B) The lower panel denotes the means \pm standard deviation of 10 experiments for each condition as determined by relative densitometry. Con, control; DAPI, 4'-6-diamidino-2-phenylindole.
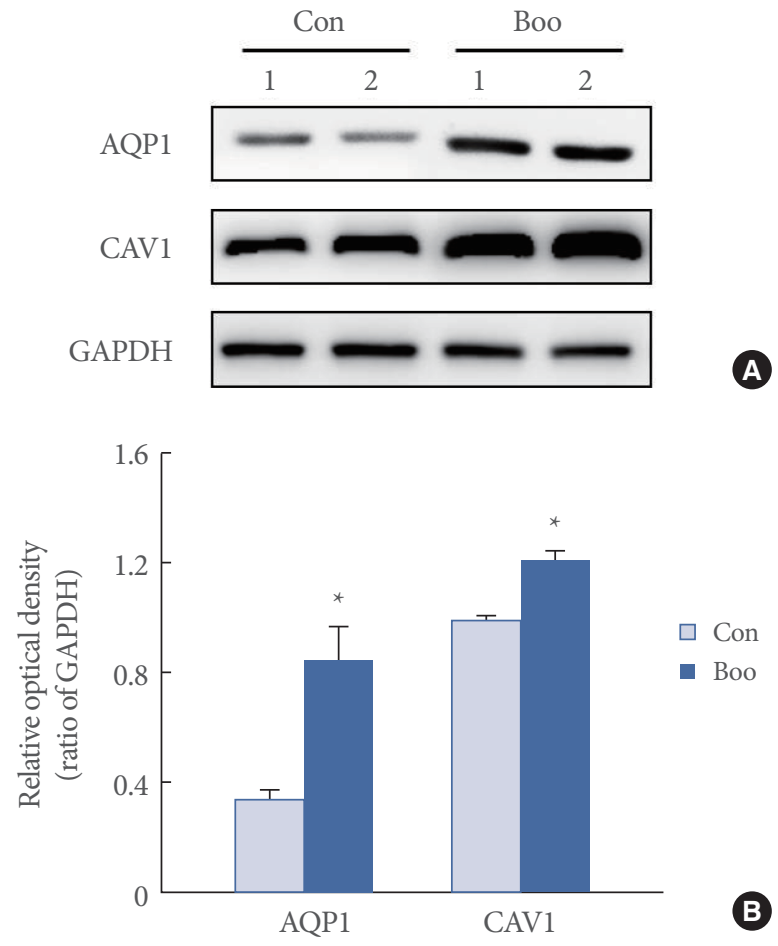

Fig. 3. (A) Immunoblotting for aquaporin 1 (AQP1) and caveolin 1 (CAV1) in the rat urinary bladder. The anti-AQP1 antibody recognizes the $27-29 \mathrm{kDa}$ bands that correspond to glycosylated AQP1. The anti-CAV1 antibody recognizes a $22 \mathrm{kDa}$ band. The anti-GAPDH antibody recognizes a $42 \mathrm{kDa}$ band. The expression of the AQP1 and CAV1 proteins was significantly increased in the bladder outlet obstruction (BOO) group. (B) The lower panel denotes the means \pm standard deviation of 10 experiments for each condition, as determined by densitometry relative to GAPDH. Con, control; GAPDH, glyceraldehyde 3-phosphate dehydrogenase. ${ }^{\star} \mathrm{P}<0.05$.

bility, and suggest that AQP1 and CAV1 might be closely associated with each other and play a role in the bladder dysfunction induced by BOO. To the best of our knowledge, this is the first study to show the possible occurrence of signaling in the suburothelial microvasculature via an interaction between AQP1 and CAV1 in bladder dysfunction induced by BOO.

Many studies have suggested that the urothelium can mediate water and solute transport under certain conditions $[17,18]$. An epithelial sodium channel $(\mathrm{ENaC})$ is known to be responsible for salt and fluid transport across the epithelia of many tissues. The role of ENaC in bladder dysfunction in male patients with clinically detected BOO has been investigated [19]. The expression level of $\mathrm{ENaC}$ was significantly greater in $\mathrm{BOO}$ patients than in controls, and their correlation was evident in the 
patients' storage symptom scores [19]. AQPs are a family of intrinsic membrane proteins that function as water channels in many cell types involved in fluid transport [8]. Until now, however, data regarding the expression of AQPs in the urinary bladder has been limited. Previously, we investigated the effect of $\mathrm{BOO}$ on the expression of AQP2-3 and nitric oxide synthase (NOS) isoforms in the rat urothelium [14]. We reported that detrusor overactivity induced by $\mathrm{BOO}$ causes a significant increase in the expression of AQP2-3, endothelial NOS, and neuronal NOS in the rat urinary bladder, suggesting that $\mathrm{AQPs}$ and NOS isoforms have a functional role in BOO-associated bladder dysfunction [14].

CAV1, a structural protein of caveolae, plays a role in the regulation of signal transduction pathways [11]. The CAV1 knockout mouse has been proposed as a novel animal model exhibiting primary hypocontraction of the urinary bladder and detrusor overactivity $[13,20]$. Loss of CAV1 is associated with the disruption of muscarinic cholinergic activities, which leads to impaired detrusor smooth muscle contraction [21]. Mice lacking CAV1 in the bladder exhibit several urologic problems such as a decreased contractility after stimulation with carbachol $[13,22]$. Bladder strips of CAV1 knockout mice generated via genetic ablation of caveolae were found to be associated with a 70\% decrease in acetylcholine release from bladder nerve terminals [13]. However, the functional significance of these organelles in relation to the micturition response is not fully understood. Cristofaro et al. [23] studied the functional effects of cholesterol depletion on specific agonist-induced bladder contraction and reported that CAV plays a central role in the regulation of G-protein-coupled signaling pathways involved in bladder smooth muscle contraction. Decreased numbers of caveolae were detected in the hypertrophied bladder smooth muscle induced by $\mathrm{BOO}$, highlighting the dynamic physiologic nature of caveolae in the urinary bladder and their possible contribution to alterations in signal transduction pathways that regulate bladder contraction [24].

However, to date, no study has investigated the relationship between AQPs and CAVs in the suburothelium of the urinary bladder or the changes in the functional activity of these proteins in response to BOO. However, a close functional interaction between AQP1 and CAV1 has been shown to regulate microvascular and alveolar epithelial permeability in the rat lung. Gao et al. [25] reported that CAV1 knockdown by small interfering RNA resulted in increased microvascular and epithelial permeability and pulmonary edema, suggesting that CAV1 plays an important role in regulating pulmonary permeability by modifying AQP1. Our results suggest that BOO may lead to a significant up-regulation of AQP1 and CAV1 expression in the rat urinary bladder, providing presumptive evidence that these proteins play a role in BOO-induced lower urinary tract symptoms, probably via modification of specific cellular transmission and signaling pathways specifically in the suburothelial microvasculature. It has been demonstrated that a decrease in blood supply to the urinary bladder occurs after BOO or bladder ischemia [26]. One possible reason for the influence of $\mathrm{BOO}$ on AQP1 and CAV1 expression is the localization of these proteins. The microvasculature is highly dependent on $\mathrm{BOO}$ and increasing intravesical pressure [26].

A limitation of this study is that the precise functional relationship between AQP1 and CAV1 could not be fully understood. Nonetheless, for the first time, we demonstrated the coexpression of AQP1 and CAV1 in the rat urinary bladder and their possible relationship with each other in the BOO rat model. However, we could not demonstrate actual functional changes in bladder activities by showing water or solute movement or signal changes. Further studies are needed to investigate the expression and localization of all subtypes of AQPs and CAVs in the urinary bladder and their functional role in the underlying mechanisms of urinary bladder function. Understanding the relationship between AQP1 and CAV1 would provide insights into the cellular mechanisms of detrusor overactivity and may suggest a new target for the pharmacological treatment of this complex urologic symptom.

In conclusion, this study showed that detrusor overactivity induced by BOO leads to increased expression of AQP1 and CAV1, which were coexpressed in the suburothelial microvasculature of the rat urinary bladder. This finding suggests that AQP1 and CAV1 might be closely related to bladder signaling activity and may have a functional role in BOO-associated detrusor overactivity.

\section{CONFLICT OF INTEREST}

No potential conflict of interest relevant to this article was reported.

\section{ACKNOWLEDGEMENTS}

This research was supported by the Basic Science Research Program through the National Research Foundation of Korea (NRF) 
funded by the Ministry of Education, Science and Technology (2012-2139, 2013-2109) and by grant CRI13005-1, Chonnam National University Hospital Research Institute of Clinical Medicine.

\section{REFERENCES}

1. de Groat WC. The urothelium in overactive bladder: passive bystander or active participant? Urology 2004;64(6 Suppl 1):7-11.

2. Birder LA, de Groat WC. Mechanisms of disease: involvement of the urothelium in bladder dysfunction. Nat Clin Pract Urol 2007; 4:46-54.

3. Chang S, Hypolite JA, Mohanan S, Zderic SA, Wein AJ, Chacko S. Alteration of the PKC-mediated signaling pathway for smooth muscle contraction in obstruction-induced hypertrophy of the urinary bladder. Lab Invest 2009;89:823-32.

4. Scott RS, Uvelius B, Arner A. Changes in intracellular calcium concentration and P2X1 receptor expression in hypertrophic rat urinary bladder smooth muscle. Neurourol Urodyn 2004;23:361-6.

5. Kim SO, Song SH, Hwang EC, Oh KJ, Ahn K, Jung SI, et al. Changes in aquaporin $(\mathrm{AQP}) 2$ and $\mathrm{AQP} 3$ expression in ovariectomized rat urinary bladder: potential implication of water permeability in urinary bladder. World J Urol 2012;30:207-12.

6. Kim SO, Song SH, Hwang EC, Park KS, Kwon DD, Ahn KY, et al. The expression of AQP1 and eNOS in menopausal rat urinary bladder. Int Neurourol J 2010;14:78-85.

7. Kim SO, Song SH, Ahn K, Kwon D, Park K, Ryu SB. Changes in aquaporin 1 expression in rat urinary bladder after partial bladder outlet obstruction: preliminary report. Korean J Urol 2010;51:281-6.

8. Verkman AS. More than just water channels: unexpected cellular roles of aquaporins. J Cell Sci 2005;118(Pt 15):3225-32.

9. Matsuzaki T, Tajika Y, Ablimit A, Aoki T, Hagiwara H, Takata K. Aquaporins in the digestive system. Med Electron Microsc 2004; 37:71-80.

10. Gannon BJ, Warnes GM, Carati CJ, Verco CJ. Aquaporin-1 expression in visceral smooth muscle cells of female rat reproductive tract. J Smooth Muscle Res 2000;36:155-67.

11. Smart EJ, Ying Ys, Donzell WC, Anderson RG. A role for caveolin in transport of cholesterol from endoplasmic reticulum to plasma membrane. J Biol Chem 1996;271:29427-35.

12. Razani B, Lisanti MP. Caveolin-deficient mice: insights into caveolar function human disease. J Clin Invest 2001;108:1553-61.

13. Lai HH, Boone TB, Yang G, Smith CP, Kiss S, Thompson TC, et al. Loss of caveolin-1 expression is associated with disruption of muscarinic cholinergic activities in the urinary bladder. Neurochem Int
2004;45:1185-93.

14. Kim SO, Choi D, Song SH, Ahn KY, Kwon D, Park K, et al. Effect of detrusor overactivity on the expression of aquaporins and nitric oxide synthase in rat urinary bladder following bladder outlet obstruction. Can Urol Assoc J 2013;7:E268-74.

15. Kim SO, Song SH, Lee SC, Cho KA, Kim HS, Hwang IS, et al. Effects of estrogens on the expression of caveolin-1 in the urinary bladders of female rats. Int Neurourol J 2011;15:13-8.

16. Kim SO, Song SH, Lee SC, Cho KA, Yu HS, Hwang IS, et al. Expression of caveolin-1 in rat urinary bladder with cyclophosphamide-induced cystitis. Int Neurourol J 2012;16:169-74.

17. Negrete HO, Lavelle JP, Berg J, Lewis SA, Zeidel ML. Permeability properties of the intact mammalian bladder epithelium. Am J Physiol 1996;271(4 Pt 2):F886-94.

18. Cross WR, Eardley I, Leese HJ, Southgate J. A biomimetic tissue from cultured normal human urothelial cells: analysis of physiological function. Am J Physiol Renal Physiol 2005;289:F459-68.

19. Araki I, Du S, Kamiyama M, Mikami Y, Matsushita K, Komuro M, et al. Overexpression of epithelial sodium channels in epithelium of human urinary bladder with outlet obstruction. Urology 2004;64: 1255-60.

20. Woodman SE, Cheung MW, Tarr M, North AC, Schubert W, Lagaud $\mathrm{G}$, et al. Urogenital alterations in aged male caveolin-1 knockout mice. J Urol 2004;171(2 Pt 1):950-7.

21. Cao G, Yang G, Timme TL, Saika T, Truong LD, Satoh T, et al. Disruption of the caveolin-1 gene impairs renal calcium reabsorption and leads to hypercalciuria and urolithiasis. Am J Pathol 2003;162: 1241-8.

22. Lai HH, Boone TB, Thompson TC, Smith CP, Somogyi GT. Using caveolin-1 knockout mouse to study impaired detrusor contractility and disrupted muscarinic activity in the aging bladder. Urology 2007;69:407-11.

23. Cristofaro V, Peters CA, Yalla SV, Sullivan MP. Smooth muscle caveolae differentially regulate specific agonist induced bladder contractions. Neurourol Urodyn 2007;26:71-80.

24. Polyak E, Boopathi E, Mohanan S, Deng M, Zderic SA, Wein AJ, et al. Alterations in caveolin expression and ultrastructure after bladder smooth muscle hypertrophy. J Urol 2009;182:2497-503.

25. Gao C, Li R, Huan J, Li W. Caveolin-1 siRNA increases the pulmonary microvascular and alveolar epithelial permeability in rats. J Trauma 2011;70:210-9.

26. Brading AF. Alterations in the physiological properties of urinary bladder smooth muscle caused by bladder emptying against an obstruction. Scand J Urol Nephrol Suppl 1997;184:51-8. 